\title{
USE OF THE PSEUDO-RIGID BODY MODEL TO SIMPLIFY THE DESCRIPTION OF COMPLIANT MICRO-MECHANISMS
}

\author{
L. G. Salmon, D. B. Gunyan \\ Integrated Microelectronics Laboratory, Department of Electrical and Computer Engineering \\ Brigham Young University \\ Provo, Utah 84602
J. M. Derderian, P. G. Opdahl, and L. L. Howell
Mechanical Engineering Department
Brigham Young University
Provo, Utah 84602

\begin{abstract}
This paper reports the use of the pseudo-rigid body method to predict the motion of compliant micro-mechanisms (CMEMS). Physical measurement of the motion of CMEMS structures is compared with predictions of the pseudo-rigid body model and finite element analysis. It is shown that for some structures the computationally simple pseudo-rigid body model can accurately predict the motion of these structures with error of less than the measurement accuracy of $0.5 \mu \mathrm{m}$ for deflections as large as $30 \mathrm{pm}$. This paper also discusses the use of the pseudo-rigid body model to provide a hierarchical approach to CMEMS design that sharply reduces the computation time required to predict the motion of complex CMEMS structures.
\end{abstract}

\section{INTRODUCTION}

The use of compliant mechanisms for the construction of MEMS provides numerous advantages over the use of conventional rigid-body structures. Compliant micro-mechanisms can be easier to fabricate, have a simpler topology, and require no assembly. [1] Compliant mechanism have no joints, can be fabricated using a single mask leveh, and its motion is dependent on materials parameters and dimensions rather than critical tolerances and friction.

The use of compliant mechanisms in MEMS technology is limited, however, because their motion is highly non-linear and thus difficult to simulate. Linear equations are often inaccurate and non-linear finite element analysis is often too computation intensive. The linear deflection equations such as the Bemoulli-Euler equation do not adequately describe the motion of compliant mechanisms because deflection in compliant micro-mechanisms typically exceed the angular deflection appropriate for these approximations. Non-linear finite element analysis can be used to accurately predict the non-linear motion of compliant micro-mechanisms, but the computation time required to describe complex structures is often prohibitive.

\section{THE PSEUDO-RIGID BODY MODEL}

Recently a new formalism has been developed to simplify description of compliant mechanisms. [2] The pseudo-rigid-body (PRB) method sharply reduces the complexity of compliant mechanism simulation by modeling the compliant mechanism as a parameterized rigid-body mechanism. The complex non-linear motion of CMEMS structure can then be predicted using simple, computationally efficient kinematic methods.
Compliant mechanisms gain some or all of their motion from the deflection of flexible members. In general, the deflections of flexible members in compliant mechanisms are large, and non-linear analysis methods must be employed to accurately predict their behavior. Early efforts in compliant mechanism analysis involved using elliptic integral solutions to model simple mechanisms. [3,4] More recently numerical methods, such as nonlinear large-deflection finite element methods, have been used to analyze the behavior of compliant mechanisms. [5]

Theory has recently been developed that simplifies the nonlinear analysis of compliant mechanisms. Before the advent of compliant mechanism analysis theory, the difficulty associated with non-linear analysis often limited the use of compliant mechanisms to very simple tasles. For example, to use non-linear finite element analysis, a designer must already have obtined a detailed initial design before the mechanism could be modeled In contrast, the pseudo-rigid-body model in compliant mechanism theory serves as a fast and efficient method of evaluating many different trial designs to meet the specific design objectives. Once a design is obtained such that it meets the specified design objectives, it may be validated or further refined using methods such as non-linear finite element analysis.

One of the key concepts in compliant mechanism theory is the pseudorigid-body model. This model provides a means of simplifying the nonlinear analysis associated with large deflections. It also is valuable in predicting the behavior of complex systems of flexible members. Pseudo-rigid-body models have been developed for a number of types of flexible segments, two of which are briefly described here as prototypes for other more complex compliant structures. If a system has flexural segments with lengths small relative to those of the rigid segments, the flexural segments are modeled as pin joints with torsional springs to represent the member stiffness, as shown in Figure 1a. [6] These segments are called small-length flexural pivots. The spring constant is calculated as:

$$
K=\frac{E I}{l}
$$

where $E$ is the modulus of elasticity, $I$ the moment of inertia, and $l$ the length of the flexible segment .

Figure $1 \mathrm{~b}$ illustrates the pseudo-rigid-body model for a long flexible segment with an end-force load. Again, the model consists of two rigid links, connected by a "characteristic pivot" to represent the displacement, and a torsional spring to model the beam resistance to the 
applied force [2]. This model predicts a deflection path of the beam that is within $0.5 \%$ of the closed-form elliptic integral solutions for quite large deflections. The location of the characteristic pivot is specified by $\gamma$, where the length of the pseudo-rigid link is $\gamma L$. A nondimensionalized "stiffness coefficient", $K_{\theta}$, is used to specify the stiffness of the torsional spring as:

$$
K=\gamma K_{\Theta} \frac{E I}{L}
$$

where $L$ is the length of the flexible segment (Figure $1 \mathrm{~b}$ ). Values of $\gamma=0.85$, and $\gamma K_{\Theta}=2.16$ are used for the constant-force mechanisms described herein. Figure 1c illustrates a similar model for fixed-guided flexible segments [7].
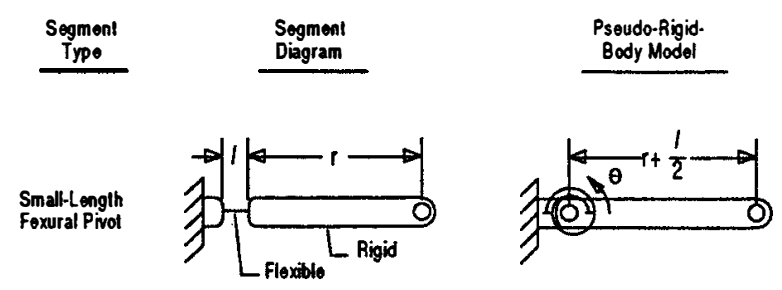

(a)

End-Force Loaded Sogment

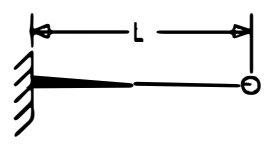

(b)
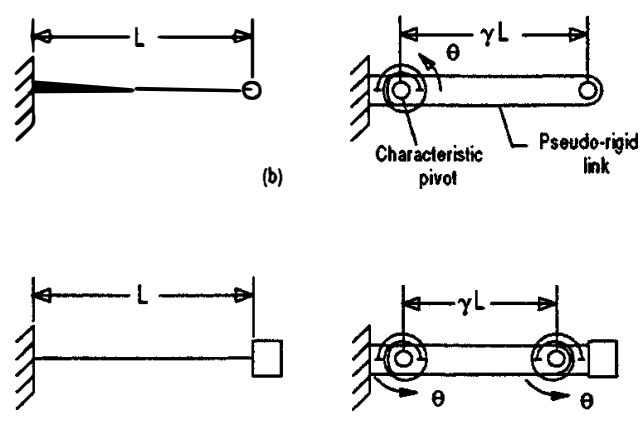

Figure 1. Pseudo-Rigid-Body models for various flexible segments.

The psendo-rigid-body models for individual flexible segments may be used to model more complex systems that include such members. This provides the ability to develop a pseudo-rigid-body model of a compliant mechanism, and then use the large body of knowledge available in the field of rigid-body mechanism analysis and design. In this way, the pseudo-rigid-body model concept acts to unify compliant mechanism and rigid-body mechanism theories.

\section{THE PARALLEL-GUIDING MECHANISM}

In order to validate use of the PRB model to describe compliant micromechanisms, we fabricated several types of CMEMS structures, measured their deflection characteristics, and compared their motion with predictions made by the $\mathrm{PRB}$ model. In this paper we report on the resuls obtained from a compliant structure that is a combination of two of the connected constant end angle segments illustrated in Figure $1 \mathrm{c}$ ). This structure is called a parallel-guiding mechanism and was chosen because it is simple enough to be modeled using non-linear finite element analysis, complex enough to be poorly modeled by use of the Bernoulli-Euler equations, and robust enough to measure deflection
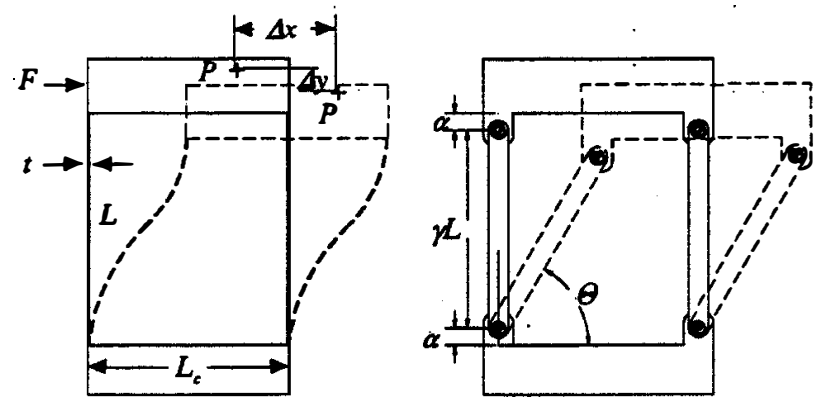

Figure 2. A compliant parallel-guiding mechanism and its pseudo-rigid-body model.

into the non-linear regime.

Figure 2 is a sketch of a prototype compliant parallel-guiding mechanism together with the equivalent PRB model representation. As can be seen from the figure, the motion of the compliant parallel-guiding mechanism can be described using the PRB model and standard kinematic position analysis. The path equations for a point " $P$ " in the compliant parallel-guiding mechanism are:

$$
\Delta x=\gamma L \cos (\theta)
$$

$$
\Delta Y=\gamma L(\sin \theta-1)
$$

$$
\alpha=\frac{L(1-\gamma)}{2}
$$

where the characteristic radius factor, $\gamma$, is determined from the orientation of the input force.

Using the stiffness coefficient, $K_{\odot}$ and the PRB equivalent model for the compliant parallel-guiding mechanism we find:

$$
E-\frac{8 K_{\theta} E I\left(\frac{\pi}{2}-\theta\right)}{L^{2} \sin \theta}
$$

where the applied force, $\mathrm{F}$, is the horizontal forcing acting on the coupler. Displacement values calculated from these equations were used to compare with displacement measurements from fabricated compliant parallel-guided micro-mechanisms and the results are discussed in the next section of the paper.

\section{EXPERIMENTAL VERIFICATION OF THE PSEUDO-RIGID BODY MODEL}

Two processes were used to fabricate the structures used in this study. The first was a single level poly-silicon process with $4 \mu \mathrm{m}$ of polysilicon and a $2 \mu \mathrm{m} \mathrm{SiO}{ }_{2}$ release layer. The second process was the MUMPS process provided by $\mathrm{MCNC}$ with $2 \mu \mathrm{m}$ of poly-silicon and a $2 \mu \mathrm{m} \mathrm{SiO}{ }_{2}$ release layer. An SEM photograph of a fabricated structure is shown in Figure 3. 


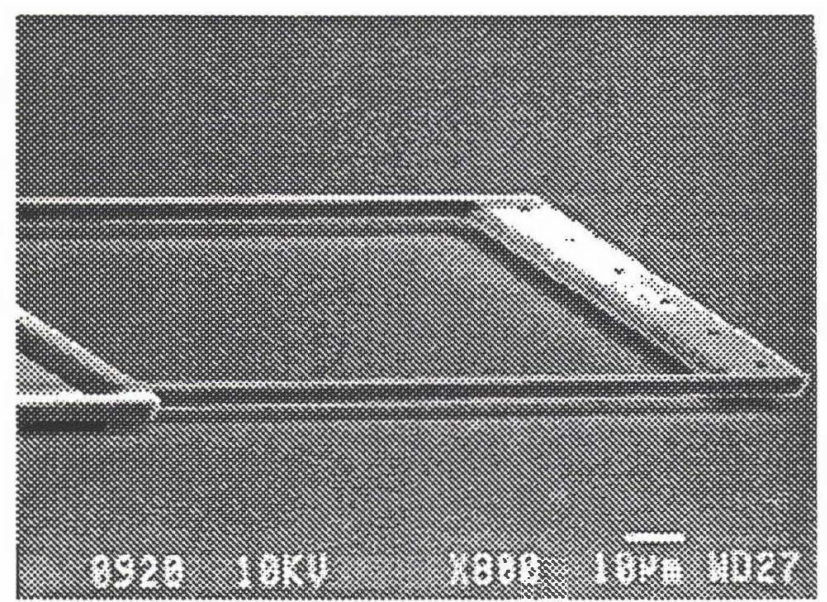

Figure 3. SEM micrograph of fabricated parallel-guiding mechanism.

Table 1 indicates the dimensions of the two mechanisms used in this work.

Table 1. List of the physical parameters for the two parallel-guided mechanisms measured in this study.

\begin{tabular}{|c|c|c|c|c|}
\hline Structure & $\mathrm{L}$ & $\mathrm{t}$ & $\mathrm{L}_{\mathrm{c}}$ & \\
\hline $\mathrm{A}$ & $100 \mu \mathrm{m}$ & $3 \mu \mathrm{m}$ & $103 \mu \mathrm{m}$ & \\
\hline $\mathrm{B}$ & $100 \mu \mathrm{m}$ & $3 \mu \mathrm{m}$ & $53 \mu \mathrm{m}$ & \\
\hline
\end{tabular}

Figures 4 and 5 compare the measured deflection of parallel mechanism $A$ and $B$ with predicted displacement extracted from the PRB model and non-linear finite element analysis using ANSYS. Data for several instances of each structure are represented in the graph. Data for

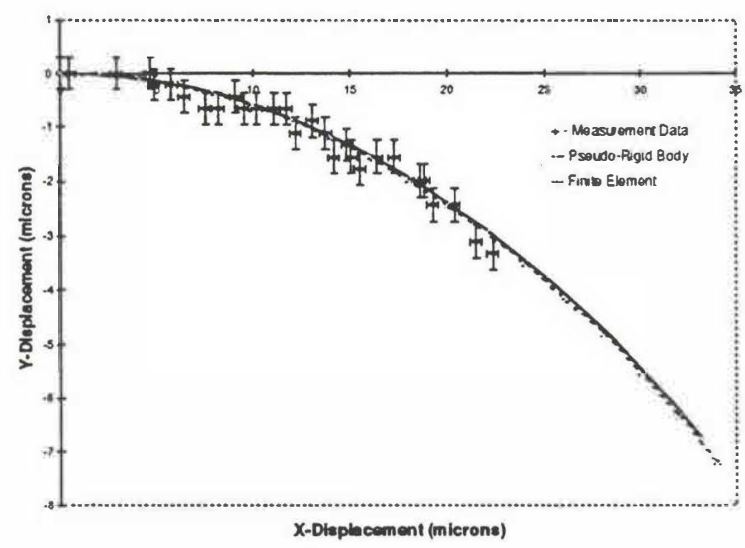

Figure 4. Graph of measured and predicted $x$ - and $y$ displacement for parallel-guided mechanism $B$.

greater deflection was not measured because of mechanism failure. The emor bars represent the uncertainty due to the pixel size of the digitized image used to determine position of the mechanism.

Both graphs show excellent correlation between measured data and predictions from the PRB model and the non-linear finite element analysis. The data from mechanism $A$ with a longer coupler length is somewhat closer to the predicted values than mechanism B, but the data from both structures closely match predicted values.

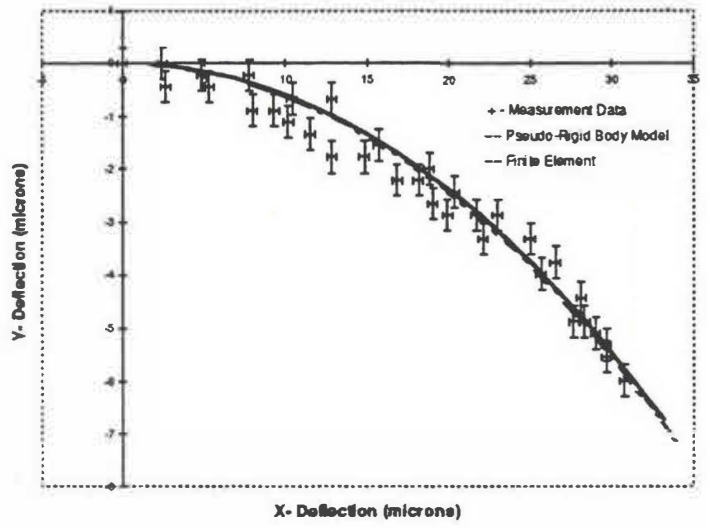

Figure 5. Graph of measured and predicted $x$ - and $y$-displacement for parallel-guided mechanism A.

The Bernoulli-Euler equations would predict zero vertical deflection, and the prodicted path is coincident with the $x$-axis. This is an accurate approximation for small, linear deflections, but the error increases as the deflection increases.

The close correlation between predicted and measured data indicates that little or no torsion is occurring in the measured structures. Torsion is a major problem with analysis of the path of CMEMS because all of the analysis assumes that the displacement of the structures is restricted to the plane of the wafer and measurement of out-of-plane displacement is difficult to determine. We measured other types of CMEMS structures and found that torsional movement severely compromised measurement data. We are currently fabricating small linewidth devices using thicker poly-silicon films in order to minimize torsional effects.

\section{CONCLUSIONS}

The close correlation between the measured data and the pseudo-rigid body predictions for parallel-guiding CMEMS mechanisms indicates the accuracy of the PRB method for moderately complex CMEMS structures. The comparatively short computation time required to simulate structures using the PRB method indicate great promise for simulation of complex CMEMS structures.

In future work we will verify the accuracy of the PRB approach to model more complex CMEMS mechanisms and to develop a formalism for complex CMEMS designs. These designs will include precise movement and bistable CMEMS mechanisms.

\section{ACKNOWLEDGMENTS}

The authors express their appreciation to the staff of the Integrated Circuit Laboratory for their assistance in fabricating the structures reported in this work. We also appreciate the fabrication efforts of MCNC in processing the wafers fabricated using the MUMPS process. This work is supported by the National Science Foundation under grant No. ECS-9528238. 


\section{REFERENCES}

1. G.K. Ananthasuresh, S. Kota, and Y. Gianchandani, "A Methodical Approach to the Design of Compliant Micromechanisms," Technical Digest of the 1994 IEEE Solid-State Sensor and Actuator Workshop, Hilton Head Isl., SC, June 1994. pp. 189-192.

2. L. L Howell and A. Midha, "Parametric Deflection Approximations for End-Loaded Large-Deflection Beams in Compliant Mechanisms," ASME Journal of Mechanical Design, Vol. 116, 1994. pp. 156-165.

3. Burns, R.H., and Crossley, F.R.E., "Kinetostatic Synthesis of Flexible Link Mechanisms," ASME Paper No. 68-Mech-36, 1968.

4. Shoup, T.E., and McLaman, C.W.,"On the Use of the Undulating Elastica for the Analysis of Flexible Link Devices," Journal of Engineering for Industry, Trans. ASME, 1971, pp. 263-267.

5. Her, I., Midha, A., and Salamon, B.A., "A Methodology for
Compliant Mechanisms Design: Part II-Shooting Method and Application," Advances in Design Automation, DE-Vol. 44-2, 18th ASME Design Automation Conference, 1992, pp. 39-45.

6. Howell, LL and Midha, A.,"A Method for the Design of Compliant Mechanisms with Small-Length Flexural Pivots," ASME Journal of Mechanical Design, Vol. 116, No. 1, 1994, pp. 280-290.

7. Howell, L.L., Midha, A, and Norton, T.W., "Evaluation of Equivalent Spring Stiffness for Use in a Pseudo-Rigid-Body Model of Large-Deflection Compliant Mechanisms," ASME Journal of Mechanical Design, Vol. 118, No. 1, 1996, pp. 126-131. 